lower at start of ADA (ExtOligo)

higher at start of ADA (ERA)

Joints with active arthritis

erythrocyte sedimentation rate (ESR)
Absence in anamnesis (PersOligo)

Shorter at start of ADA (ERA), longer at start of ADA (PersOligo) lower at start of ADA (ERA)

lower at start of ADA (PersOligo) response to adalimumab treatment in 12 months in patients with different JIA category.

Methods: Analysis include patients with enthesitis-related arthritis (ERA, $n=56$ ), RF-negative polyarthritis (polyRF-, $n=50$ ), extended oligoarthritis (extOligo, $n=30$ ), and persistent oligoarthritis (persOligo, $n=62$ ) with median age 10.5 (IQR 7-14) and median JADAS-71 19.5 (IQR 15-28). Patients were divided to response groups after 12 month treatment with ADA according to ACRPedi criteria, achieving inactive disease by Wallace criteria and JADAS-71 cut-off point as excellent, intermediate and poor responders. For each of JIA category univariate and multivariate logistic regression analysis was conducted to identify potential baseline factors associated with treatment response. Baseline factors included clinical, laboratory and anamnestic data.

Results: ADA was shown to be effective in all groups with $90 \% / 89 \% / 82 \% / 63 \%$ children with ACR30/50/70/90 during one year therapy. The most significant factors $(p<0.05)$ associated with response to ADA treatment are presented in summarized table.

Our findings demonstrated that different predictors corresponded with different JIA categories. Interestingly subjective scales of disease activity was shown to be strongly associated with response to therapy. At the same time VAS severity at baseline were inversely correlated with achievement of good response. Duration of morning stiffness correlated with excellent response in children with 2 different categories. However, for ERA patients shorter duration was associated with better response to treatment while vice versa for PersOligo patients.

Conclusions: Predictors of response to ADA treatment differ in JIA categories. Low disease activity parameters (clinical and laboratory) at baseline not always predict good response to therapy.

Disclosure of Interest: E. Kashchenko Grant/research support from: Novartis, E. Alexeeva Grant/research support from: Roche, Abbott, Pfizer, Bristol-Myers Squibb, Centocor, Novartis, Speakers bureau: Roche, Merck Sharp \& Dohme, Abbott, Bristol-Myers Squibb, Medac, Novartis, Pfizer, T. Bzarova Grant/research support from: Roche, Pfizer, Novartis, Speakers bureau: Roche, Merck Sharp \& Dohme, Abbott, Pfizer, S. Valieva Grant/research support from: Roche, BristolMyers Squibb, Speakers bureau: Roche, Merck Sharp \& Dohme, Bristol-Myers Squibb, Medac, Novartis, R. Denisova Grant/research support from: Roche, Centocor, Novartis, Speakers bureau: Roche, Merck Sharp \& Dohme, Abbott, Medac, O. Lomakina: None declared, K. Isaeva Grant/research support from: Roche, Novartis, M. Soloshenko: None declared, A. Karaseva: None declared DOI: 10.1136/annrheumdis-2017-eular.4011

\section{AB0952 EXPERIENCE OF TOCILIZUMAB USE IN TREATMENT OF JUVENILE IDIOPATHIC ATRTRITIS IN CHELYABINSK REGIONAL CHILDREN HOSPITAL}

G.A. Glazyrina ${ }^{1}$, N.A. Kolyadina ${ }^{2}$. ${ }^{1}$ South Ural Governmental Medical University; ${ }^{2}$ Chelyabinsk Regional Children Hospital, Chelyabinsk, Russian Federation

Background: Recently due to application of interleukin (IL)-6 inhibitors prognosis for systemic juvenile idiopathic arthritis (SJIA) has significantly improved. 526 children with JIA are under monitoring in Chelyabinsk region, 42 children have SJIA. Tocilizumab is the drug of choice for SJIA treatment. It is registered within the Russian Federation for use in children older than 2 years. In Chelyabinsk Regional Children Hospital tocilizumab has been used for 5 years.

Methods: 18 children (12 boys, 6 girls) aged from 2 to 17 (mean age 10.7 years) diagnosed with SJIA were under monitoring. Disease duration was from 6 months to 15 years (mean duration 6 years). JIA was diagnosed based on ILAR diagnostic criteria. SJIA was diagnosed in 16 children, sero-negative polyarthritis was diagnosed in 2 children. Tocilizumab was introduced intravenously every 2 or 4 weeks in dose of $12 \mathrm{mg} / \mathrm{kg}$ for children $<30 \mathrm{~kg}$ or $8 \mathrm{mg} / \mathrm{kg}$ for children $\geq 30 \mathrm{~kg}$. Therapy duration was from 3 months to 5 years (average duration 23 months). Assessment of disease activity and therapy efficiency was conducted in accordance with ACR pedi criteria. Nonparametric statistical methods were used to compare results.

Results: Prior to tocilizumab use high disease activity was observed in all children. Average number of joints with active arthritis was 13,5 [6;15] (Me [25;75\%]). Average number of joints with functional impairments $-12,5[6 ; 15]$. Average ESR (according to Panchenkov) - 50 [40;60]mm/h, CRP 98,6 [55;139]g/L. Assessment of functional activity according to CHAQ questionnaire- 2,09 [2;2,5]. Activity assessment according to VAS by doctor - 82 [75;90]. Assessment of parents according to VAS 84 [80;90]. No eye lesions were found in children under monitoring. All 16 children with SJIA had fever, hepatosplenomegaly and lymphadenopathy, the rash had 11 children, polyserositis - 8. In 5 children there was a complication in the form of a syndrome of macrophage activation.

During the tocilizumab therapy a decrease in disease activity was observed in all patients. Mean number of joints with active arthritis was 1 [0;2] (Me $[25 ; 75 \%])(P=0.0002)$. Mean number of joints with functional impairments -3 $[0 ; 3](P=0.0003)$. Average ESR was $4[3 ; 5] \mathrm{mm} / \mathrm{h}(P=0.00002)$, CRP $0,75[0 ; 1] \mathrm{g} / \mathrm{L}$ $(P=0.0003)$. Assessment of functional activity according to $C H A Q$ questionnaire was $0,25[0 ; 0.5](P=0.0004)$. Activity assessment according to VAS by doctor - $16[10 ; 20](P=0.0002)$. Assessment of parents according to VAS $18[10 ; 20]$ $(\mathrm{P}=0.0002)$.

Clinical disease remission (according to ACR pedi criteria- $\geq 90 \%$ ) was observed in 11 patients after 6-9 months of treatment. Remission duration up to now is from 3 months to 4 years. Efficiency according to ACR pedi criteria is $70 \%$ in 6 children, $50 \%$ in 1 .

The drug was well-tolerated. Undesirable effect such as allergic skin reactions were observed only in one child. In one child the lack of efficiency produced by switching to canakinumab. Drug was cancelled in 3 patients due to long-term remission (3-4 years), but 2 of them after a year needed the resumption of therapy in connection with the aggravation of the disease.

Conclusions: Tocilizumab therapy was highly effective and safe in patients with JIA. Clinical remission was achieved in $61,1 \%$ children. Decrease in disease activity was observed in $39,9 \%$ of children. No serious undesirable effects were reported.

Disclosure of Interest: None declared

DOI: 10.1136/annrheumdis-2017-eular.3354

\section{AB0953 ARTHRITIS FOLLOWING PARASITIC INFECTION IN THE DIFFERENTIAL DIAGNOSIS OF JUVENILE IDIOPATHIC ARTHRITIS}

M. Maschio ${ }^{1}$, S. Pieropan ${ }^{2}$, F. Caldonazzi ${ }^{1}$, G. Dallagiacoma ${ }^{2}$, V. Ravagnani ${ }^{3}$, D. Degani ${ }^{1}$, G. Piacentini ${ }^{1}$, D. Biasi ${ }^{2}$, M. Rossini ${ }^{2} .{ }^{1}$ UOC pediatria; ${ }^{2}$ UOC reumatologia, AOUI, Verona; ${ }^{3}$ Reumatologia, Azienda Ospedaliera Carlo Poma, Mantova, Italy

Background: According to literature arthritis is a rare complication following parasitic infection. In the italian pediatric population parasitic infection is manly caused by Enterobius Vermicularis; it is often asymptomatic but common symptoms can be anal nocturnal itching, insomnia, irritability, abdominal pain together with arthralgia

Objectives: To describe cases of mono or poliarthritis due to parasitic infection in young patients followed at the Verona's Pediatric Rheumathology Clinic from 2010 to 2016

Methods: Medical records of 8 patients followed at the Pediatric Rheumatology were analyzed. The mean age of 8 patients was 8 (F:M 3:5). The following data were evaluated: anamnesis data, clinical symptoms (anal itching, irritability, arthralgia, abdominal pain and arthritis), Blood test and scotch tape test. All patients underwent joint ultrasound (US). Physical symptoms of joint's involvement were evaluated in all children by an experienced rheumatologist.

Results: 6 patients had symmetrical poliathirtis, 2 patients had monoarthritis (knee and hand). Symptom's complaint were systemic (12,5\%), abdominal (25\%), and general pruritus (25\%). Blood test confirmed high inflammation indices $(25 \%)$ and hypereosinophilia (12,5\%).Serological tests and stool investigations allowed to diagnose the following infections: Enterobius vermicularis (6 cases) giardia lamblia (1 case); dientaomeba fragilis (1 case).Joint US evidenced synovium hyperplasia in $75 \%$ of the cases and tenosynovitis in $50 \%$ of the cases.After appropriate antiparasitic treatment complete articular and systemic symptomatic remission was observed (100\% cases); also joint US control normalized. 2 cases, after about 6 months, were re-evaluated for arthritis relapse and in both cases a parasitic reinfection was confirmed.

Conclusions: Analysis of this series of patients underlines the following data: arthritis can be a manifestation of parasitic infection; treatment must be aimed against the parasite involved in order to achieve complete clinical and laboratory data remission; reinfection must always be considered in cases of relapse; differential diagnosis of this form of arthritis with other chronic polyarthritis is fundamental due to the risk of disseminated infection in case of immunosuppressive treatment

Disclosure of Interest: None declared

DOI: 10.1136/annrheumdis-2017-eular.4876 